\title{
Actividad repelente de aceites esenciales contra las picaduras de Lutzomyia migonei (Diptera: Psychodidae)
}

\author{
Elsa Nieves ${ }^{1 *}$, Janett Fernández Méndez ${ }^{1}$, José Lias ${ }^{2}$, Maritza Rondón ${ }^{1} \&$ Benito Briceño ${ }^{3}$ \\ 1. LAPEX, Departamento de Biología, Facultad de Ciencias, Universidad de Los Andes, Mérida-Venezuela; \\ nevelsa@ula.ve, Janett12@hotmail.com,rmaritza@ula.ve \\ 2. Laboratorio de Productos Naturales, Jardín Botánico, UNELLEZ, Barinas-Venezuela; liasj@ula.ve \\ 3. Laboratorio de Botánica, Departamento de Biología, Facultad de Ciencias, Universidad de Los Andes, Mérida- \\ Venezuela; bricenob@ula.ve \\ * Correspondencia.
}

Recibido 03-XII-2009. Corregido 14-VI-2010. Aceptado 15-VII-2010.

\begin{abstract}
Repellent activity of plant essential oils against bites of Lutzomyia migonei (Diptera: Psychodidae). Natural repellents from plant extracts have demonstrated good efficacy against bites of some insect species. The present study evaluated the repellent effect of essential oils extracted from 8 plants species against bites of Lutzomyia migonei, the Leishmania vector. The essential oils were extracted by steam destillation in Clevenger chamber, from the following plants: Hyptis suaveolens, Pimenta racemosa, Piper marginatum, Monticalia imbricatifolia, Pseudognaphalium caeruleocanum, Espeletia shultzii, Plecthranthus amboinicus and Cinnamomun zeylanicum. Repellency tests were performed under laboratory conditions by the human hand method in cage assays, using female colonies of L. migonei. The more effective oils were tested at variable concentrations on different volunteers. The protection percentage and time were calculated. The results showed what oils of P. caeruleocanum and C. zeylanicum were the most effective. Although P. amboinicus oil also had repellent effect showed an irritant effect. The oils $P$. marginatum, H. suaveolens and $P$. racemosa showed no repellent effect, while the rest of oil extracts showed significant repellency in variable degrees. P. caeruleocanum and $C$. zeylanicum oils provided the $95 \%$ protection against bites of $L$. migonei for $3 \mathrm{~h}$. The P. caeruleocanum oil showed the greatest protection time, with a mean over $4 \mathrm{~h}$ and $3 \mathrm{~h}$ at concentrations of $50 \%$ and $10 \%$ respectively. The results suggest that the P. caeruleocanum oil could represent a potential natural repellent against Leishmania vectors. Rev. Biol. Trop. 58 (4): 1549-1560. Epub 2010 December 01.
\end{abstract}

Key words: repellent, sand flies, leishmaniasis, essential oils, Lutzomyia migonei.

La leishmaniasis es un grupo de enfermedades causadas por protozoarios parásitos del género Leishmania (Kinetoplastida: Trypanosomatidae), que es transmitida a los humanos y animales por la picadura de insectos dípteros hematófagos de la familia Psychodidae (Neuber 2008). A nivel mundial, la leishmaniasis constituye la tercera enfermedad parasitaria. Los incrementos en la transmisión mundial de esta enfermedad, se deben principalmente, al aumento de la transmisión peri-doméstica, producto de la adaptación de los flebótomos a estas áreas, y promovida a su vez, por la deforestación, urbanización y cambios climáticos en las zonas de transmisión (Alvar et al. 2006, Reithinger et al. 2007).

En la actualidad, no se dispone de un esquema inmunoprofiláctico eficaz para prevenir las infecciones por Leishmania. La quimioterapia con antimoniales pentavalentes, continúa siendo, desde hace más de 50 años, la manera convencional más rápida, práctica y eficaz para el tratamiento de la leishmaniasis (Maroli \& Khoury 2004, Neuber 2008). Sin embargo, estas drogas tienen alta toxicidad y costos elevados. Además, recientemente se 
han presentado casos de resistencia en algunas especies de Leishmania, por lo que es necesario desarrollar alternativas de control contra la leishmaniasis. Una vacuna podría constituir una medida preventiva muy eficiente, sin embargo, los estudios realizados no han superado la fase de prueba (Ampuero et al. 2005, Dujardin 2006).

El control vectorial mediante el rociamiento de insecticidas de origen químico, especialmente a nivel peri domiciliar e intra domiciliar, así como también, a nivel selvático y la utilización de mosquiteros impregnados de los mismos, es una de las medidas generalmente implementadas, aunque su éxito depende de la conducta de los flebótomos (Alexander \& Maroli 2003, Maroli \& Khoury 2004, SirakWizeman et al. 2008). A pesar de lo práctica y atractiva que resulta esta medida, presenta los siguientes inconvenientes que pueden ser limitantes para el control de los flebótomos: desconocimiento de la bio-ecología y los criaderos de los insectos vectores, la aparición de resistencia, discontinuidad de los programas anti vectoriales, sus altos costos, que han afectado la salud humana, animal y la biosfera en general (Thavara et al. 2001, Kroeger et al. 2002, Katz et al. 2008, Sharma \& Singh 2008).

El control de los flebótomos con insecticidas no es tan viable, una manera de solventar dicho problema, es desarrollar repelentes, como medida alternativa de protección contra los vectores (Alexander \& Maroli 2003). Los repelentes, si bien no matan al insecto, son una buena alternativa preventiva para evitar la picadura de los vectores. Entre los ingredientes activos de los repelentes contra la picadura de los insectos, se incluye el DEET ( $N$ - $N$-dietil3-metilbenzamida), el cual continúa siendo el líder del mercado desde hace 50 años. A pesar de que el DEET ha superado muchas pruebas de toxicidad, presenta un alto grado de absorción cutánea y hay pruebas que muestran su efecto tóxico desde leves a severos niveles (Alexander et al. 1995, Tawatsin et al. 2001, Katz et al. 2008). Por lo anterior, es que se ha tratado de sustituir por repelentes sintéticos como el IR3535, SS220, Picaridin y MGK-326 en diferentes formulaciones y concentraciones, con actividad por más de 6h (Thavara et al. 2001, Naucke et al. 2006, Muller et al. 2008). Estas sustancias fueron probadas contra los flebótomos Phlebotomus mascitii Grassi, $P$. dubosqui Neveu-Lemaire y Lutzomyia longipalpis Luzt \& Neiva, con resultados satisfactorios (Muller et al 2008, Sirak-Wizemam et al. 2008).

Sin embargo, es necesario implementar alternativas de control contra las picaduras de los flebótomos que sean menos riesgosas y contaminantes. Las plantas medicinales y en particular sus metabolitos secundarios, son una buena opción efectiva como repelente contra los insectos (Maroli \& Khoury 2004, Erler et al. 2006). Se ha probado una variedad de plantas como repelentes naturales contra mosquitos, de las cuales existen reportes para citronela (Melissa officinalis), cedro (Cedrus), eucalipto (Eucaliptus), pimienta (Pimenta), limón (Citrus), geranio (Geranium), andiroba (Carapa), melisa (Melissa) y semillas de soya (Glycine); el tiempo de protección de estas especies varió de 2 a $30 \mathrm{~min}$. de protección total y cerca de $90 \mathrm{~min}$. para el aceite de semillas de soya (Tawatsin et al. 2001, Fradin \& Day 2002, Maroli \& Khoury 2004, Katz et al. 2008).

Una de las plantas más utilizadas contra insectos es el árbol de neem (Azadirachta indica Jussieu) como antialimentación, antioviposición, regulador de crecimiento y repelente (Rajkumar \& Jebanesan 2005). Esta planta también tiene actividad repelente satisfactoria contra flebótomos (Sharma \& Dhiman 1993; Srinivasan \& Kalyanasundaram 2001, Andrade-Coelho et al. 2006, 2009).

Por otra parte, Valerio \& Maroli (2005) reportaron protección efectiva contra las picaduras del flebótomo P. papatasi Scopoli, utilizando aceite de ajo (Alium sativum Linnaeus). Resultados similares se han obtenido con el aceite de las hojas de Citrus medica Linnaeus con actividad repelente contra mosquitos y flebótomos (Rojas \& Scorza 1990, Rojas 1999). También se ha ensayado la actividad repelente contra las picaduras de flebótomos 
de los aceites esenciales de citronela, linalol y geraniol en velas, encontrándose un $24 \%, 55 \%$ y $79 \%$ de protección, respectivamente (Muller et al. 2008). Sin embargo, el geraniol, usado en placas evaporadoras, no produce un efecto repelente contra $P$. papatasi y $P$. sergenti Parrot (Sirak-Wizeman et al. 2008).

Pocos son los estudios de repelentes botánicos contra los vectores de Leishmania en comparación con los realizados con mosquitos (Das et al. 2003, Amora et al. 2009). Actualmente se sabe que Lutzomyia migonei França es una especie antropofílica de amplia distribución en el continente Americano, incriminada como transmisora de leishmaniasis cutánea en la región de los Andes Venezolanos, Brasil y recientemente incriminada como vector de leishmaniasis visceral en Argentina (Nieves \& Pimenta 2000, Salomon et al. 2010). Por lo anterior, en el presente estudio se investiga la actividad repelente de los aceites esenciales extraídos de ocho especies de plantas medicinales, contra las picaduras de L. migonei en humanos, en condiciones experimentales.

\section{MATERIALES Y MÉTODOS}

Aceites esenciales: Los aceites esenciales fueron extraídos de Hyptis suaveolens Linnaeus (mastranto), Pimenta racemosa Mill (bay-rum), Piper marginatum Jacq (anisillo), Plectranthus amboinicus Lour (oreganón), Cinnamomun zeylanicum Blume (canela), recolectadas en el Jardín Botánico de la Universidad Experimental de Los Llanos Ezequiel Zamora, Barinas-Venezuela, ubicado al noroeste de la ciudad de Barinas, al pie de la Cordillera de los Andes; a una altura de $200 \mathrm{msnm}$ y presentando una temperatura media anual de $28^{\circ} \mathrm{C}$. Además se utilizaron Pseudognaphalium caeruleocanum Steyermark (vira-vira), Espeletia shultzii Weddell (frailejón) y Monticalia imbricatifolia Schults; recolectadas en el Páramo de Piñango, ubicado en el estado Mérida-Venezuela, a una altura de $3900 \mathrm{msnm}$ y temperatura media anual entre $\operatorname{los} 12$ y $15^{\circ} \mathrm{C}$. Las plantas fueron recolectadas entre diciembre y abril, de cultivos silvestres en floración, las cuales se identificaron y se depositaron en el Herbario MERF, Facultad de Farmacia y Bioanálisis, Universidad de Los Andes (ULA), Estado Mérida y en el Herbario de la Universidad Experimental de Los Llanos Ezequiel Zamora (UNELLEZ), Estado Barinas, Venezuela. Los aceites esenciales se obtuvieron a partir de 1 a $2 \mathrm{~kg}$ de hojas frescas por hidrodestilación, utilizando la trampa de Clevenger, obteniendo entre 0.8 y $3.8 \mathrm{ml}$ y se almacenaron bajo refrigeración $\left(-4^{\circ} \mathrm{C}\right)$ hasta su uso, por un periodo no mayor de cuatro meses.

Flebótomos: Se utilizaron un total de 1680 hembras de L. migonei de 5 a 7 días de edad, provenientes de una colonia de laboratorio, iniciada con ejemplares capturados en la localidad Arenal a una altura de 1360 m.s.n.m., ubicada en Ejido, Mérida, Venezuela $\left(8^{\circ} 35^{\prime}\right.$ ' N-71 ${ }^{\circ}$ ' O). Las colonias se mantienen utilizando la técnica descrita por Killick-Kendrick et al. (1977) en incubadora a $25^{\circ} \mathrm{C} \pm 1$ y a $80 \% \pm 10$ de humedad relativa, en el Laboratorio de Parasitología Experimental de la Universidad de Los Andes (LAPEX-ULA), Mérida, Venezuela.

Ensayos: Los ensayos se realizaron mediante la prueba de la mano en la jaula (Schreck \& McGovern 1989, WHO 1996). Para cada ensayo, se emplearon jaulas de tela nylon de $15 \times 15 \times 15 \mathrm{~cm}$. Antes de cada ensayo las manos de los voluntarios se lavaron con jabón sin olor y abundante agua. Para cada uno de los ensayos, se aplicó $50 \mu \mathrm{l}\left(0.416 \mu \mathrm{l} / \mathrm{cm}^{2}\right)$ del aceite esencial puro $(100 \%)$ para ensayar sobre la superficie del dorso de la mano derecha, desde la muñeca hasta la punta de los dedos, luego de 30 min de la aplicación del aceite se introdujo la mano en la jaula, que contenía a 20 hembras hambrientas de L. migonei. Los ensayos se realizaron durante un periodo de $20 \mathrm{~min}$ de exposición de la mano, con intervalos de 30min de descanso, hasta que ocurriera la primera y segunda picadura del insecto. Seguidamente, se registraron el total de picadas del último período de exposición. Para cada aceite se estimó el tiempo de protección y para los aceites que presentaron efecto repelente satisfactorio (tiempo de protección $>180 \mathrm{~min}$, sin efecto 
secundarios), se determinó el porcentajes de repelencia (Tawatsin et al. 2001, Fradin \& Day 2002, Rajkumar \& Jebanesan 2005). Los aceites puros ensayados que presentaron efecto repelente satisfactorio, se probaron con distintos voluntarios y en concentraciones de $50 \%$ y $10 \%$, en aceite mineral enriquecido con Aloe vera y Vitamina $\mathrm{E}$ de procedencia comercial marca Johnson ${ }^{\circledR}$.

El tiempo de protección, corresponde, al tiempo que trascurre desde la aplicación del producto hasta que ocurra la segunda picadura (Schreck \& Mc Govern 1989, Thavara et al. 2001, Das et al. 2003).

Las picaduras de cada mano de los voluntarios se cuantificaron y el porcentaje de protección (PP) se calculó de acuerdo con la fórmula:

$$
P P=\frac{N c-N t}{N c} \times 100
$$

donde $N c=$ número de picaduras en la mano control en determinado período de tiempo, $N t=$ número de picaduras en la mano tratada en el mismo período (Rueda et al. 1998, Frances et al. 2005).

Como control se utilizó la mano izquierda con aceite mineral comercial marca Jhonson ${ }^{\circledR}$. Como control positivo se utilizó repelente comercial DEET (15\%) marca OFF® y como negativo la mano sin producto, procediéndose de manera similar como se indicó con los aceites a ensayar.

Para cada prueba se realizaron ensayos por triplicado, tres ensayos por semana, para un total de 81, cada uno con diferentes grupos de flebótomos.

Los voluntarios fueron tres mujeres de raza mestiza, piel morena, edades entre de $30-50$ años y peso entre $55-75 \mathrm{~kg}$, sin historia clínica de reacciones alérgicas a las picaduras de insectos, miembros del Laboratorio de Parasitología Experimental de la Universidad de Los Andes LAPEX-ULA, quienes accedieron voluntariamente a los ensayos (y con firma de consentimiento). Los ensayos se realizaron cumpliendo las normas éticas de la Universidad de Los Andes, Mérida, Venezuela.
Todos los ensayos se llevaron a cabo en condiciones ambientales similares para cada uno, sin corriente de viento, humedad relativa entre $60-70 \%$, temperatura entre $24-26^{\circ} \mathrm{C}$ y en oscuridad.

Los resultados se analizaron por la prueba no paramétrica de Mann-Whitney de dos vías, para comparar la repelencia de cada aceite; y por el análisis de Kruskal Wallis para evaluar diferencias entre voluntarios y concentraciones, utilizando el paquete estadístico SPSS. Un valor $\mathrm{p} \leq 0.05$ se consideró estadísticamente significativo.

\section{RESULTADOS}

Los resultados mostraron una media de $8.48 \mathrm{~min}$. para la primera picadura (p1) y de 13.69 min. para la segunda picadura (p2) de $L$. migonei sobre voluntarios sin la aplicación de ningún producto.

Los aceites esenciales de $P$. caeruleocanum y $C$. zeylanicum presentaron la mayor actividad repelente contra las picaduras de L. migonei en condiciones de laboratorio, para una media del tiempo de protección de 301.12min ( $>5 \mathrm{~h}$ ) y $251.82 \mathrm{~min}$ ( $>4 \mathrm{~h}$ ) (Fig. 1). El aceite de $P$. amboinicus presentó efecto repelente satisfactorio, $251.36 \mathrm{~min}(>4 \mathrm{~h})(\mathrm{p}=0.04)$, pero ocasionó irritación, picazón y enrojecimiento en la piel. Los aceites esenciales de E. shultzii y M. imbricatifolia mostraron baja actividad repelente para un tiempo de protección de $32.46 \mathrm{~min}$ $(<1 \mathrm{~h})$ y de $87.1 \mathrm{~min}(>1 \mathrm{~h})$. Mientras que el resto de los aceites esenciales, de P. marginatum, $H$. suaveolens y $P$. racemosa no mostraron protección con relación a los controles $(\mathrm{p}<0.05)$.

El aceite de $P$. caeruleocanum y $C$. zeylanicum revelaron un $95 \%$ de protección contra las picaduras de L. migonei a $3 \mathrm{~h}$ (Fig. 2). Es importante resaltar que el aceite de $P$. caeruleocanum ocasionó un efecto "Knock-down" del $10 \%$ de la densidad de hembras por ensayo.

Con relación a la evaluación de diferentes concentraciones de los aceites de las dos especies de plantas con efecto repelente significativo, se encontró para el aceite esencial de $P$. caeruleocanum una disminución progresiva 


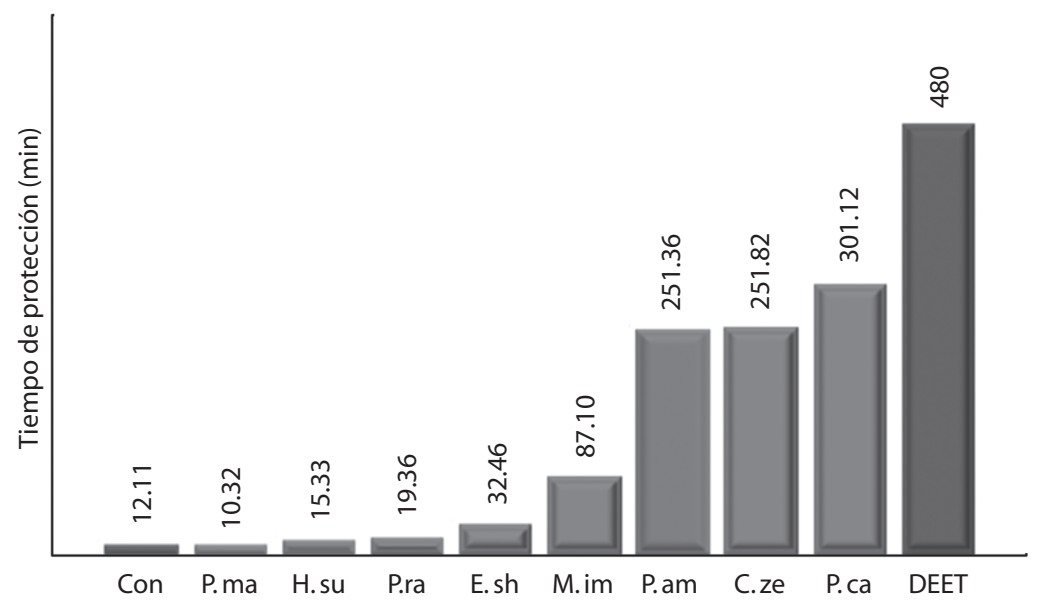

Fig. 1. Tiempo de protección de los aceite esenciales al 100\% ensayados contra las picaduras de L. migonei. Con=Control, P. ma=Piper marginatum, H. su=Hyptis suaveolens, $\mathrm{P} . \mathrm{ra}=$ Pimenta racemosa, $\mathrm{E} . \mathrm{sh}=$ Espeletia shultzii, $\mathrm{M}$. im=Monticalia imbricatifolia, P. am=Plectranthus amboinicus, C. ze=Cinnamomun zeylanicum, $\mathrm{P} . \mathrm{ca}=$ Pseudognaphalium caeruleocanum y DEET $=N$ - $N$-dietil-3-metilbenzamida.

Fig. 1. Protection time of the essential oils test against the bites of L. migonei. Con= Control, P. ma=Piper marginatum, H. su=Hyptis suaveolens, P. ra=Pimenta racemosa, E. sh=Espeletia shultzii, M. im=Monticalia imbricatifolia, P. am=Plectranthus amboinicus, $C$. ze=Cinnamomun zeylanicum, $\mathrm{P} . \mathrm{ca}=$ Pseudognaphalium caeruleocanum and DEET=N- $\mathrm{D}$ diethyl-3-methylbenzamide.

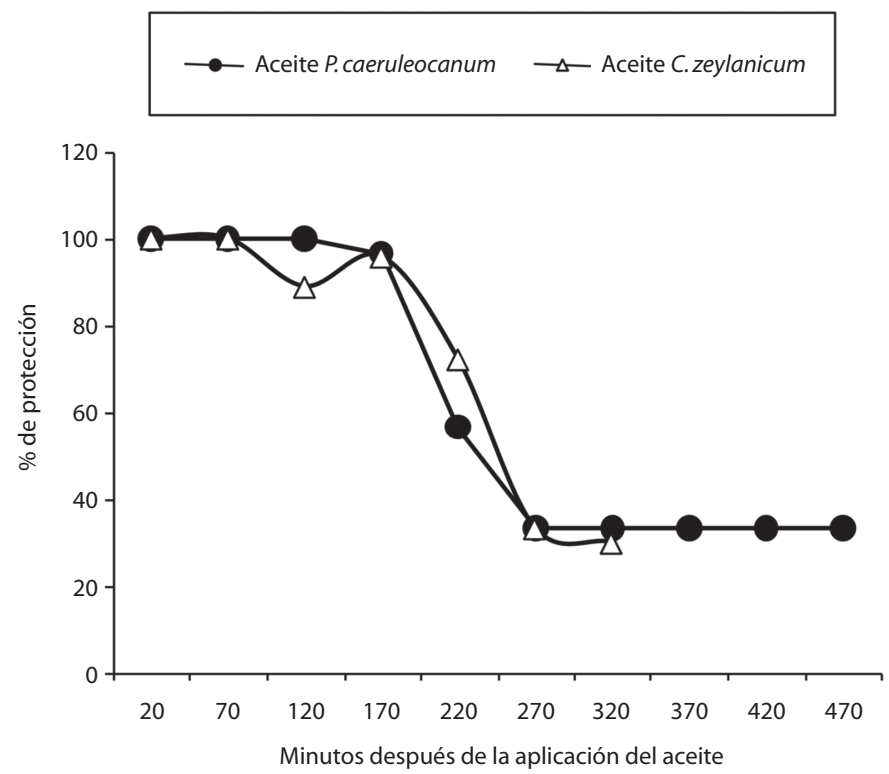

Fig. 2. Porcentaje de protección de los aceites esenciales de P. caeruleocanum y C. zeylanicum contra las picaduras de $L$. migonei.

Fig. 2. Protection percentage of the oils of P. caeruleocanum and C. zeylanicum against the bites of L. migonei. 
significativa $(\mathrm{p}=0.043)$ del tiempo de protección que va desde un 301.12min $(\sim 5: 30 \mathrm{~h})$ al $100 \%$, de $250.81 \mathrm{~min}$ al $50 \%(\sim 4 \mathrm{~h})$ y de $164.99 \mathrm{~min}(\sim 3 \mathrm{~h})$ al $10 \%$ (Fig. 3). Mientras que con el aceite esencial de $C$. zeylanicum se observó una disminución drástica del tiempo de protección, de $251.82 \mathrm{~min}(>4 \mathrm{~h})$ para el $100 \%$, de $158.85 \mathrm{~min}(<3 \mathrm{~h})$ al $50 \%$ y de apenas $13.83 \mathrm{~min}$ al $10 \%$, en esta última concentración no hubo efecto repelente en relación a los controles ( $\mathrm{p}=0.337$ ) (Fig. 3).

La evaluación del tiempo de protección del aceite esencial de $P$. caeruleocanum con los distintos voluntarios no mostró diferencia significativa ( $\mathrm{p}=0.692$ ) (Fig. 4). Mientras que con el aceite esencial de $C$. zeylanicum si se observó diferencia significativa ( $\mathrm{p}=0$.009) (Fig. 4).

\section{DISCUSIÓN}

Varias especies de plantas pertenecientes al género Pseudognaphalium (Asteraceae), se han utilizado en la medicina tradicional como antisépticos locales, en enfermedades bronquiales y gastrointestinales, así como repelente contra insectos (Cáceres et al. 1990, Mendoza et al. 1997, Urzúa 2004). El efecto repelente del aceite esencial de P. caeruleocanum (100\%) obtenido en el presente trabajo contra las picaduras de L. migonei mostró el mayor efecto repelente con un tiempo de protección de $4 \mathrm{~h}$. Sin embargo, su eficiencia fue menor en comparación al repelente comercial DEET (control positivo), que más que repelente, actúa como insecticida, gracias a su efecto "Knock-down" sobre L. migonei. Es importante resaltar que el aceite de $P$. caeruleocanum (100\%), también ocasionó un efecto "Knock-down" en un 10\%, sobre L. migonei. P. caeruleocanum es una planta endémica de los Andes Venezolanos empleada por los habitantes del páramo merideño para el tratamiento de diversas afecciones respiratorias (Badillo 1996). También, se le ha reportado actividad bactericida, por inhibir el desarrollo de bacterias grampositivas como Staphylococcus aureus Rosenbach y Enterococcus feacalis Andrews y Horder (Fernández et al. 2008). En un estudio reciente sobre

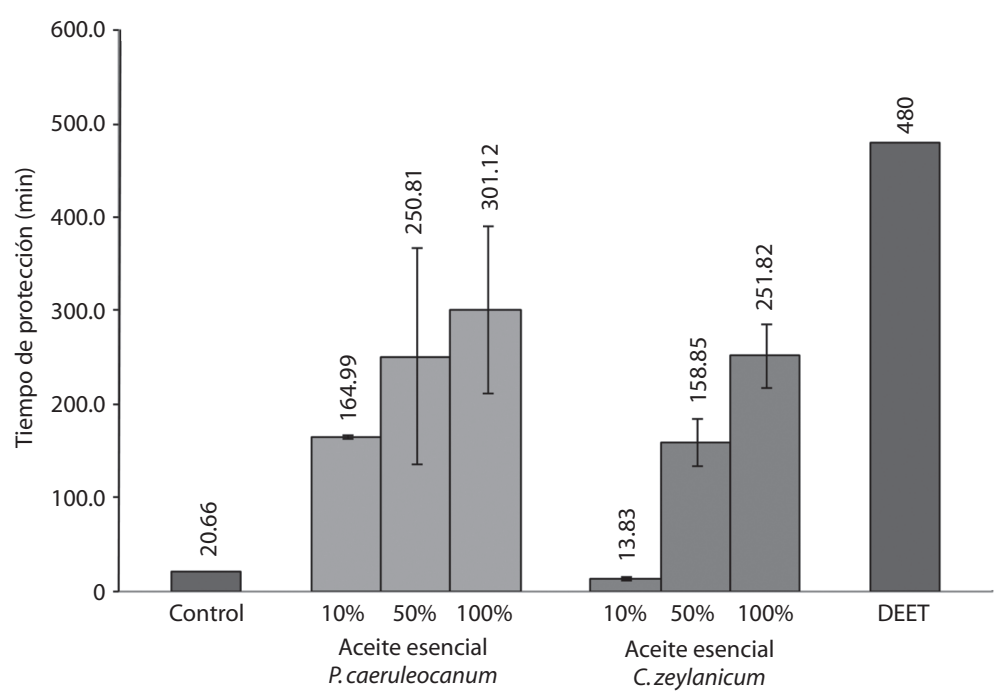

Fig. 3. Tiempo de protección de los aceites esenciales de P. caeruleocanum y C. zeylanicum a diferentes concentraciones contra las picaduras de L. migonei.

Fig. 3. Protection time of the oils of P. caeruleocanum and C. zeylanicum with different concentrations against the bites of L. migonei. 


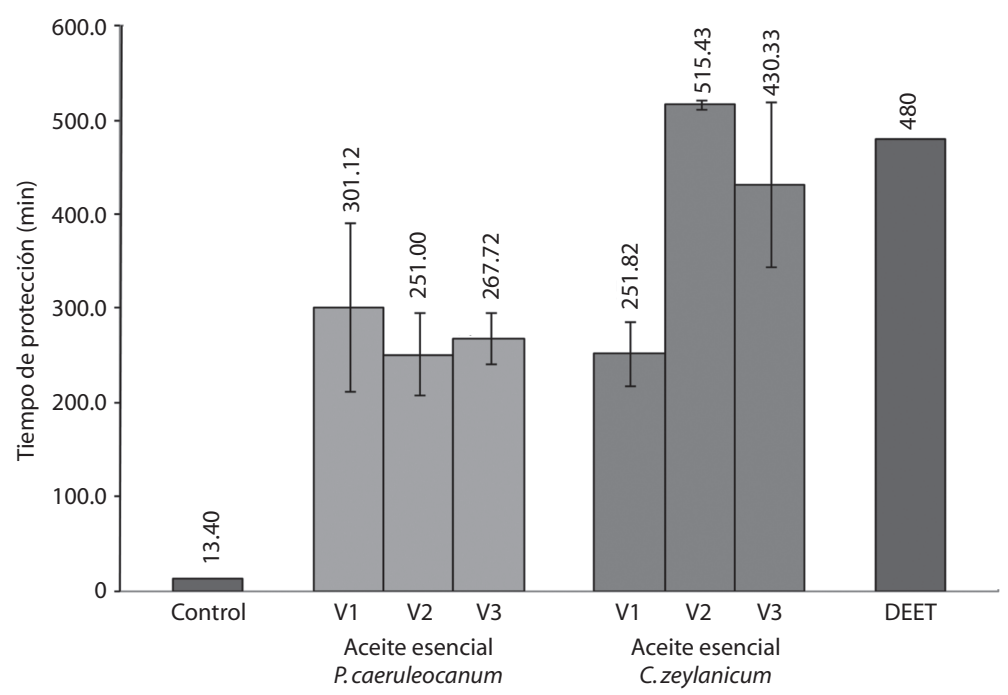

Fig. 4. Tiempo de protección de los aceites esenciales de P. caeruleocanum y C. zeylanicum con distintos voluntarios contra las picaduras de L. migonei.

Fig. 4. Protection time of the oils of P. caeruleocanum and C. zeylanicum with different volunteers against the bites of $L$. migonei.

composición química del aceite esencial de $P$. caeruleocanum, se identificaron 24 componentes, los compuestos principales fueron $\delta$ - 3 careno, trans- $\beta$-ocimeno, $\beta$-pineno y nerolidol (Fernández et al. 2008).

Otro resultado alentador de repelencia contra las picaduras de L. migonei lo mostró $C$. zeylanicum. El aceite esencial de C.zeylanicum posee alta actividad repelente contra Anopheles stephensi Liston, Aedes. aegypti Linnaeus y Culex quinquefasciatus Say (Prajapati et al. 2005). También se ha reportado su eficacia contra larvas y adultos de A. pharoensis Theobald y larvas de Musca domestica Linnaeus, bajo condiciones controladas de laboratorio (Halim 2008). Al comparar el tiempo de protección de los aceites de $P$. caeruleocanum y $C$. zeylanicum con los repelentes naturales comercializados contra mosquitos, tales como el aceite de citronela y el de eucalipto (30min a $1 \mathrm{~h}$ ), se encontró que los aceite $P$. caeruleocanum y $C$. zeylanicum protegieron por más tiempo contra las picaduras de L. migonei. Trongtokit et al. (2005) reportaron menos de 120min del tiempo de protección para 38 aceites esenciales de plantas ensayados contra las picaduras de varias especies de mosquitos a concentraciones de 10 o 50\%. Lamentablemente, no existen suficientes estudios con repelentes botánicos contra flebótomos que permitan hacer comparaciones sobre su aplicación en humanos en condiciones de laboratorio (Sharma \& Singh 2008, Amora et al. 2009). Lo anterior, quizás producto de la falta de disponibilidad de ejemplares por las dificultades en la cría de los flebótomos en el laboratorio (Yaghoobi-Ershadi et al. 2006). Además, los datos obtenidos en otros estudios, no son comparables, como los reportados en ensayos de campo. Incluso, la comparación directa de los resultados no es adecuada, debido a discrepancias entre los protocolos, especies utilizadas y estimación del tiempo de protección (Masetti 2006). Por otro lado, la evaluación del repelente se debe realizar preferiblemente con humanos, ya que los ensayos con animales o membranas artificiales, pudieran no representar lo que ocurre cuando se aplican en la piel humana (Trongtokit et al. 2005). 
Los aceites esenciales de E. shultzii y $M$. imbricatifolia, mostraron baja actividad repelente. E. shultzii es una planta herbácea medicinal de la familia Asteraceae, endémica de los páramos venezolanos conocida como frailejón. $\mathrm{Su}$ aceite esencial es rico en hidrocarburos monoterpénicos (Castrillo 2006, Ibañez \& Usubillaga 2006). M. imbricatifolia (Asteraceae), es otra planta medicinal de los páramos venezolanos conocida popularmente como romerito, que ha sido poco estudiada (Briceño \& Morillo 2002) y no se conocen reportes sobre alguna actividad repelente.

A pesar de que los aceites de $P$. marginatum, $H$. suaveolens y P. racemosa, no evidenciaron efecto repelente contra L. migonei, existen reportes de actividad repelente de estos aceites sobre otros insectos. El aceite esencial de las hojas de $P$. marginatum (Piperaceae), es usado en la medicina popular como tonificante y diurético. Además, como repelente contra picaduras de insectos y cobras, se le considera como larvicida de mosquitos y con actividad cercaricidal contra las cercarias de Schistosoma mansoni Sambon (Trematoda). Se le reportan 40 componentes químicos, los principales compuestos son, asarona, safrol, $\beta$-ocimeno, $\gamma$-terpineno y alcohol patchouli (Frischkorn et al. 1978, Autran et al. 2009). Mientras que $H$. suaveolens, familia Lamiaceae, se utiliza tradicionalmente como una planta repelente contra insectos (Seyoum et al. 2002). El aceite reduce significativamente la actividad de alimentación de $A$. aegypti, siendo los principales componentes volátiles reportados el $\beta$-cariofileno, bergamoteno, terpinoleno, humuleno sabineno y limoneno (Jaenson et al. 2006). P. racemosa (bay rum), de la familia Myrtaceae, es una planta aromática con alto contenido de compuestos fenólicos, existen reportes de su actividad como microbicida, antiséptica y desinfectante (García et al. 2004). También, se ha utilizado como larvicida de A. aegypti y C.pipiens Linnaeus, produciendo $100 \%$ de mortalidad; se le considera como una buena candidata para el control de los mosquitos transmisores de la malaria (Lee 2006).
El aceite de $P$. amboinicus (Lamiaceae), conocida como oreganón presentó buena actividad de repelencia contra las picaduras de L. migonei, pero también mostró un efecto irritante en la piel. Esta hierba es utilizada para el tratamiento de enfermedades inflamatorias como la artritis reumática, como desinfectante, antimicótico, antimicrobiano, estimulante muscular y en el tratamiento de cálculos vesicales y renales (Fernández et al. 2003, Nogueira et al. 2008, Chang et al. 2010). Además, se le ha reportado un efecto tóxico (Vizoso et al. 1999), hallazgo que coincide con el encontrado en el presente trabajo, por lo que esta planta requiere de mayores estudios.

Poco se conoce acerca del mecanismo de acción de los repelentes, se cree que interfieren con los receptores olfatorios de atracción hacia la fuente de alimento del insecto. Cada sustancia repelente actúa según su composición y al mecanismo especie específico del insecto involucrado en la atracción hacia el hospedador. La mayoría de los ingredientes activos de los repelentes actúan como neurotoxinas o toxinas respiratorias para los insectos, bloqueando la respuesta electrofisiológica de las neuronas sensoriales olfatorias atractivas del olor, e inhibiendo el comportamiento de atracción hacia la fuente de alimento de los receptores de acido láctico y $\mathrm{CO}_{2}$, entre otros (Ditzen et al. 2008). La actividad repelente de una planta se debe principalmente a sus componentes, tales como los fenoles, terpenos o alcaloides; sin embargo, son necesarios futuros estudios para identificar su papel y los diversos mecanismos fisiológicos del insecto involucrados en la atracción hacia el hospedador (Rajkumar \& Jebanesan 2005).

Con relación al tiempo de protección de los aceites de $P$. caeruleocanum y $C$. zeylanicum, a diferentes concentraciones, se observó una disminución del tiempo de protección al disminuir las concentraciones de dichos aceites, semejante a lo que se reporta con diferentes repelentes botánicos contra mosquitos (Fradin \& Day 2002 , Erler et al. 2006).

En cuanto al tiempo de protección en distintos voluntarios, las diferencias significativas observadas con C.zeylanicum, podrían deberse 
a factores propios de cada individuo involucrado, que pueden afectar la atracción del insecto, tales como, la temperatura corporal, la exhalación de $\mathrm{CO}_{2}$, amoníaco y otras sustancias químicas emanadas por la piel, y el tipo de piel, entre otros (Sirak-Wizeman et al. 2008, Ditzen et al. 2008). Sin embargo, el aceite esencial de $P$. caeruleocanum no presentó diferencias significativas con los distintos voluntarios, tal vez, producto de su alta volatilidad en comparación con el aceite de $C$. zeylanicum. Múltiples son los factores que determinan la eficiencia del aceite como repelente, entre ellos se incluyen la especie de insecto, su densidad y los mecanismos bioquímicos involucrados en la atracción hacia el hospedador (Fradin \& Day 2002); además, de los factores propios del aceite y del ambiente (Ditzen et al. 2008). Como lo sugiere Masetti (2006), es necesario realizar mayores estudios para comprender los factores que intervienen en la eficiencia de los repelentes. En conclusión, el aceite de $P$. caeruleocanum mostró alta eficacia de protección como repelente contra las picaduras de $L$ migonei. Los resultados sugieren que el aceite $P$. caeruleocanum podría ser un potencial candidato como repelente natural contra los transmisores de Leishmania, aunque se requiere continuar los estudios en relación a sus componentes y actividad repelente.

\section{AGRADECIMIENTO}

Los autores agradecen a Buitrago D., Morales A. y Rojas L. por su valiosa colaboración. Al CDCHT-ULA (proyecto: Cod: C-1606-08-03-B) y a la LOCTI (proyecto: CODIGO- L-C-13-07-03-MRW) por el financiamiento otorgado.

\section{RESUMEN}

Los repelentes naturales de extractos de plantas han mostrado eficacia contra diferentes especies de insectos. El presente estudio evaluó la acción repelente de aceites esenciales extraídos de ocho especies de plantas contra las picaduras de Lutzomyia migonei, vector de Leishmania. Los aceites esenciales se obtuvieron por hidrodestilación, utilizando una trampa de Clevenger, a partir de Hyptis suaveolens, Pimenta racemosa, Piper marginatum, Monticalia imbricatifolia, Pseudognaphalium caeruleocanum, Espeletia shultzii, Plectranthus amboinicus y Cinnamomun zeylanicum. Los ensayos de repelencia se realizaron sobre humanos en condiciones de laboratorio, frente a hembras de L. migonei provenientes de colonia, utilizando el método de la mano en la jaula. Los aceites con efecto repelente también se ensayaron con distintos voluntarios y concentraciones. Se determinó el porcentaje de protección y el tiempo de protección. Los resultados revelaron que el aceite de $P$. caeruleocanum y $C$. zeylanicum fueron los más efectivos. El aceite de $P$. amboinicus presentó efecto de repelencia satisfactorio, sin embargo, ocasionó picazón y toxicidad en la piel. Los aceites de $P$. marginatum, $H$. suaveolens y $P$. racemosa no evidenciaron efecto repelente; el resto de los aceites presentaron repelencia significativa en grado variable. Los aceites de $P$. caeruleocanum y C. zeylanicum mostraron un $95 \%$ de protección de 3 h contra las picaduras de L. migonei. El aceite de $P$. caeruleocanum presentó el mayor tiempo de protección, de más de $4 \mathrm{~h}$ y de $2 \mathrm{~h}$ en concentraciones de $50 \%$ y $10 \%$, respectivamente. Los resultados sugieren que el aceite $P$. caeruleocanum podría ser un potencial candidato como repelente natural contra la picadura de dípteros posibles transmisores de Leishmania.

Palabras clave: repelente, flebótomos, leishmaniasis, aceites esenciales, Lutzomyia migonei.

\section{REFERENCIAS}

Alexander, B. \& M. Maroli. 2003. Control of phlebotomine sandflies. Med. Vet. Entomol. 17: 1-18.

Alexander, B., H. Cadena, M.C. Usma \& C.A. Rojas. 1995. Laboratory and field evaluations of a repellent soap containing diethyl toluamide (DEET) and permethrin against phlebotomine sand flies (Diptera: Psychodidae) in Valle del Cauca, Colombia. Am. J. Trop. Med. Hyg. 52: 169-170.

Alvar, J., S. Yactayo \& C. Bern. 2006. Leishmaniasis and poverty. Trends Parasitol. 22: 552-557.

Amora, S.S.A., C.M.L. Bevilaqua, F.M.C. Feijo, N.D. Alves \& M.V. Maciel. 2009. Control of phlebotomine (Diptera:Ssychodidae) leishmaniasis vectors. Neotrop. Entomol. 38: 303-310.

Ampuero, J., M. Urdaneta \& V.O. Macedo. 2005. Risk factors for cutaneous leishmaniasis transmission in children aged 0 to 5 years in an endemic area of Leishmania (Viannia) braziliensis. Cad. Saúde Publica. 21: $161-170$.

Andrade-Coelho, C.A., N.A De Souza, M.D. Feder, C.E. Da Silva, G.E. De Souza, P. Azambuja, M. Salabert 
\& E.F. Rangel. 2006 Effects of azadirachtin on the development and mortality of Lutzomyia longipalpis larvae (Diptera: Psychodidae: Phlebotominae). J. Med. Entomol. 43: 262-266.

Andrade-Coelho, C.A., N.A. De Souza, C. Gouveia, V.C. Silva, M.S. Gonzalez \& E.F. Rangel. 2009. Effect of Fruit and Leaves of Meliaceae Plants (Azadirachta indica and Melia azedarach) on the Development of Lutzomyia longipalpis Larvae (Diptera: Psychodidae: Phlebotominae) Under Experimental Conditions J. Med. Entomol. 46: 1125-1130.

Autran, E.S., I.A. Neves, C.S. Da Silva, G.K. Santos, C.A. Da Câmara \& D.M. Navarro. 2009. Chemical composition, oviposition deterrent and larvicidal activities against Aedes aegypti of essential oils from Piper marginatum Jacq. (Piperaceae). Bioresour. Technol. 100: 2284-2288.

Badillo, V. 1996. Los géneros de las Compositae (Asteraceae) de Venezuela. Ernstia 6: 51-168.

Briceño, B. \& G. Morillo. 2002. Catálogo abreviado de las plantas con flores de los páramos de Venezuela. Parte I: Dicotiledóneas (Magnoliopsida). Act. Bot. Venez. 25: 23-29.

Caceres, A., O. Cano, B. Samayoa \& L. Aguilar. 1990. Plants used in Guatemala for the treatment of gastrointestinal disorders, screening of 84 plants against enterobacteria. J. Ethnopharmacol. 30: 55-73.

Castrillo, M. 2006. Photosynthesis in three altitudinal populations of the Andean plant Espeletia schultzii (Compositae). Rev. Biol. Trop. 54: 1143-1149.

Chang, J.M., C.M. Cheng, L.M. Hung, Y.S. Chung \& R.Y. Wu. 2010. Potential use of Plectranthus amboinicus in the Treatment of Rheumatoid Arthritis. Evid Based Complement Alternat. Med. 7: 115-120.

Das, N. G., P. K. Talukdar \& S. C. Das. 2003. Evaluation of botanicals as repellents against mosquitoes. J. Vet. Borne Dis. 40: 49-53.

Ditzen, M., M. Pellegrino \& L.B. Vosshall. 2008. Insect odorant receptors are molecular targets of the insect repellent DEET. Science 28: 1838-1842.

Dujardin, J. 2006. Risk factors in the spread of leishmaniases: towards integrated monitoring? Trends Parasitol. 22: 4-6.

Erler, F., I. Ulug \& B. Yalcinkaya. 2006. Repellent activity of five essential oils against Culex pipiens. Fitoterapia 77: 491-494.
Fenández, J., D. Buitrago, J. Velasco, L. Rojas \& A. Morales. 2008. Composición química y actividad antibacteriana del aceite esencial de Pseudognaphalium caeruleocanum (Steyermark) A. Anderberg. Rev. Latinoam. Quím. 36: 29-35.

Fernández, P., M. David, M.T. Buznego, R. Menéndez, M. Llanio \& H.M.P. Saad. 2003. Perfil Neurofarmacológico de Plectranthus amboinicus (Lour) Spreng. (orégano francés). Rev. Neurol. 36: 98-99.

Fradin, M, \& J. Day. 2002. Comparative efficacy of insect repellents against mosquito bites. N. Engl. J. Med. 347: 13-18.

Frances, S.P. \& R.A. Wirtz. 2005. Repellents: past, present, and future. J. Am. Mosq. Control Assoc. 4: 1-3.

Frischkorn, C.G., H.E. Frischkorn \& E. Carrazzoni. 1978. Cercaricidal activity of some essential oils of plants from Brazil. Naturwissenschaften 65: 480-483.

García, M.D., M.A. Fernández, A. Álvarez \& M.T. Saenz. 2004. Antinociceptive and anti-inflammatory effect of the aqueous extract from leaves of Pimenta racemosa var. ozua (Mirtaceae). J. Ethnopharmacol. 91: 69-73.

Halim, A.A.S. 2008. Efficacy of Zingiber officinale on third stage larvae and adult fecundity of Musca domestica and Anopheles pharoensis. J. Egypt Soc. Parasitol. 38: 385-392.

Ibañez, J. \& A. Usubillaga. 2006. The essential oil of Espeletia schultzii of different altitudinal populations. Flavour Frag. J. 21: 286-289.

Jaenson, T.G., K. Pålsson \& A.K. Borg-Karlson. 2006. Evaluation of extracts and oils of mosquito (Diptera: Culicidae) repellent plants from Sweden and GuineaBissau. J. Med. Entomol. 43: 113-119.

Katz, T.M., J.H. Miller \& A.A. Hebert. 2008. Insect repellents: historical perspectives and new developments. J. Am. Acad. Dermatol. 58: 865-871.

Killick-Kendrick, R., A.J. Leaney \& P.D. Ready. 1977. The establishment, maintenance and productivity of a laboratory colony of Lutzomyia longipalpis (Diptera: Psychodidae). J. Med. Entomol. 13: 429-440.

Kroeger, A., E.V. Avila \& L. Morrison. 2002. Insecticide impregnated curtains to control domestic transmission of cutaneous leishmaniasis in Venezuela: cluster randomised trial. British Med. J. 325: 810-813.

Lee, H.S. 2006. Mosquito larvicidal activity of aromatic medicinal plant oils against Aedes aegypti and Culex 
pipiens pallens. J. Am. Mosq. Control Assoc. 22: 292-295.

Masetti, A.S.M. 2006. Arm in cage tests to compare skin repellents against bites of Aedes albopictus. Bull. Insectol. 59: 157-160.

Maroli, M. \& C. Khoury. 2004. Prevention and control of leishmaniasis vector: current approaches. Parassitologia 46: 211-215.

Mendoza, L., M. Wilkens \& A. Urzua. 1997. Antimicrobial study of the resinous exudates and of diterpenoides and flavonoids isolated from some Chilean Pseudognaphalium (Asteraceae). J. Ethnopharmacol. 58: 85-88.

Müller, G.C., A. Junnila, V.D. Kravchenko, E.E. Revay, J. Butlers \& Y. Schlein. 2008. Indoor protection against mosquito and sand fly bites: a comparison between citronella, linalool, and geraniol candles. J. Am. Mosq. Control Assoc. 24: 150-153.

Naucke, T., S. Lorentz \& H.W. Grünewald. 2006. Laboratory testing of the insect repellents IR $3535^{\circledR}$ and DEET against Phlebotomus mascitti and P. duboscqi (Diptera: Psychodidae). International J. Med. Microbiol. 296: 230-232.

Neuber, H. 2008. Leishmaniasis. J. Dtsch Dermatol. Ges. 6: 754-765.

Nieves, E. \& P.F.P. Pimienta. 2000. Development of Leishmania (Viannia) braziliensis and Leishmania (Leishmania) amazonensis in the sand fly Lutzomyia migonei (Diptera: Psychodidae). J. Med. Entomol. 37: 134-140.

Nogueira J.C., M.F. Diniz \& E.O. Lima. 2008. In vitro antimicrobial activity of plants in Acute Otitis Externa. Braz. J. Otorhinolaryngol. 74: 118-124.

Prajapati, V., A.K. Tripathi, K.K. Aggarwal \& S.P. Khanuja. 2005. Insecticidal, repellent and oviposition-deterrent activity of selected essential oils against Anopheles stephensi, Aedes aegypti and Culex quinquefasciatus. Bioresour Technol. 96: 1749-1757.

Rajkumar, S. \& A. Jebanesan. 2005. Repellency of volatile oils from Moschosma polystachyum and Solunum xanthocarpum against filarial vector Culex quinquefasciatus Say. Trop. Biomed. 22: 139-142.

Reithinger, R., J:C. Dujardin, H. Louzir, C. Pirmez, B. Alexander \& S. Brooker. 2007. Cutaneous leishmaniasis. Lancet Infect. Dis. 7: 581-596.

Rojas, E. \& J. Scorza. 1990. Protección personal con un repelente natural contra Lutzomyia youngi, vector de leishmaniasis cutánea urbana en Venezuela. Bol. Dir. Malariol. San. Amb. 20: 1-4.

Rojas, E. 1999. Extracción y rendimiento de aceite esencial de hojas de Citrus medica con uso para la protección personal contra mosquitos transmisores. Rev. Amb. Ecol. 6: 164-178.

Rueda, L.M., L.C. Rutledge \& R.K. Gupta. 1998. Effect of skin abrasions on the efficacy of the repellent deet against Aedes aegypti. J. Am. Mosq. Control Assoc. 14: 178-182.

Salomón, O.D., M.G. Quintana, G. Bezzi, M.L. Morán, E. Betbeder \& D.V. Valdéz. 2010. Lutzomyia migonei as putative vector of visceral leishmaniasis in La Banda, Argentina. Acta Trop. 113: 84-7.

Schreck, C.E. \&. Mc Govern. 1989. Repellents and other personal protection strategies against Aedes albopictus. J. Am. Mosq. Control Assoc. 5: 247-252.

Seyoum, A., K. Palsson, S. Kung'a, E.W. Kabiru, W. Lwande, G.F. Killeen, A. Hassanali \& B.G.J. Knols. 2002. Traditional use of mosquito-repellent plants in western Kenya and their evaluation in semi-field experimental huts against Anopheles gambiae: ethnobotanical studies and application by thermal expulsion and direct burning. Trans. Roy. Soc. Trop. Med. Hyg. 96: 225-231.

Sharma, U. \& S. Singh. 2008. Insect vectors of Leishmania: distribution, physiology and their control. J. Vector Borne Dis. 45: 255-572.

Sharma, V.P. \& R.C. Dhiman. 1993. Neem oil as a sand fly (Diptera: Psychodidae) repellent. J. Am. Mosq. Control Assoc. 9: 364-366.

Sirak-Wizeman, M., R. Faiman, A. Al-Jawabreh \& A. Warburg. 2008. Control of phlebotomine sandflies in confined spaces using diffusible repellents and insecticides. Med. Vet. Entomol. 22: 405-412.

Srinivasan, R. \& M. Kalyanasundaram. 2001. Relative efficacy of DEPA and neem oil for repellent activity against Phlebotomus papatasi, the vector of leishmaniasis. J. Commu. Dis. 33: 180-184.

Tawatsin, A., S.D Wratten, R.R. Scott, U. Thavara \& Y. Techadamrongsin. 2001. Repellency of volatile oils from plants against three mosquito vectors. J. Vector Ecol. 26: 76-82.

Thavara, U., A. Tawatsin, J. Chompoosri, W. Suwonkerd, U. Chansag \& P. Asavadachanukorn. 2001. Laboratory and field evaluations of the insect repellent 3535 (ethyl butylacetylaminopropionate) and Deet against 
mosquito vectors in Thailand. J. Am. Mosq. Control Assoc. 17: 190-195.

Trongtokit, Y., Y. Rongsriyam, N. Komalamisra \& C. Apiwathnasorn. 2005. Comparative repellency of 38 essential oils against mosquito bites. Phytother. Res. 19: 303-309.

Urzúa, A. 2004. Mono and sesquiterpenes in the trichome secreted exudates from Psedognaphalium cheiranthifolium, P. heterotrichium, P. robustum. Biochem. System. Ecol. 32: 211-214.

Valerio, L. \& M. Maroli. 2005. Valutazione dell'effetto repellente ed anti-feeding dell'aglio (Allium sativum) nei confronti dei flebotomi (Diptera: Psychodidae). Ann. Ins. Super Sanitá 41: 253-256.
Vizoso, P.A., A.R. Ruiz, A.E. Armenteros, J.B. Betancourt \& M.M. Décalo. 1999. Plectranthus amboinicus (Lour) Spreng (Orégano Francés) Estudio toxicogenético de un extracto fluido y del aceite esencial. Rev. Cubana Plan. Med. 3: 68-73.

WHO. 1996. Report of the WHO informal consultation on the evaluation and testing of insecticides. CTD/ WHOPES/IC/96.1, Control of Tropical Diseases Division. World Health Organization, Geneva, 69 p.

Yaghoobi-Ershadi, M.R., A.A. Akhavan, E. Jahanifard, H. Vatandoost, G.H. Amin, L. Moosavi, A.R. ZahraeiRamazani, H. Abdoli \& M.H. Arandian. 2006. Repellency effect of Myrtle essential oil and Deet against Phlebotomus papatasi, under laboratory conditions. Iranian J. Publ. Health 35: 7-13. 